\title{
Precision tests of the Standard Model with kaon decays at CERN
}

\author{
Chris Parkinson ${ }^{* \dagger}$ \\ University of Birmingham \\ E-mail: cjp@hep.ph.bham.ac.uk
}

Recent results and prospects for precision tests of the Standard Model in kaon decay in flight experiments at CERN are presented. A measurement of the ratio of leptonic decay rates of the charged kaon at a $0.4 \%$ precision constrains the parameter space of new physics models with, for example, an extended Higgs sector. Searches for neutrino mass states and the dark photon in the $\sim 100 \mathrm{MeV} / c^{2}$ mass range based on samples collected in 2003-2007 are in progress and prospects will be discussed. The NA62 experiment starting in 2014 will search for a range of lepton number and lepton flavour violating decays of the charged kaon and the neutral pion at improved sensitivities down to $\sim 10^{-12}$, probing new physics scenarios involving, for example, heavy Majorana neutrinos. Prospects for the NA62 L0 trigger strategy for rare and forbidden decays will be discussed.

XIIth International Conference on Heavy Quarks \& Leptons 2014

25-29 August 2014

Schloss Waldthausen, Mainz, Germany

\footnotetext{
${ }^{*}$ Speaker.

†for the NA62 Collaboration: G. Aglieri Rinella, R. Aliberti, F. Ambrosino, B. Angelucci, A. Antonelli, G. Anzivino, R. Arcidiacono, I. Azhinenko, S. Balev, J. Bendotti, A. Biagioni, C. Biino, A. Bizzeti, T. Blazek, A. Blik, B. Bloch-Devaux, V. Bolotov, V. Bonaiuto, M. Bragadireanu, D. Britton, G. Britvich, N. Brook, F. Bucci, V. Buescher, F. Butin, E. Capitolo, C. Capoccia, T. Capussela, V. Carassiti, N. Cartiglia, A. Cassese, A. Catinaccio, A. Cecchetti, A. Ceccucci, P. Cenci, V. Cerny, C. Cerri, O. Chikilev, R. Ciaranfi, G. Collazuol, P. Cooke, P. Cooper, G. Corradi, E. Cortina Gil, F. Costantini, A. Cotta Ramusino, D. Coward, G. D’Agostini, J. Dainton, P. Dalpiaz, H. Danielsson, J. Degrange, N. De Simone, D. Di Filippo, L. Di Lella, N. Dixon, N. Doble, V. Duk, V. Elsha, J. Engelfried, T. Enik, V. Falaleev, R. Fantechi, L. Federici, M. Fiorini, J. Fry, A. Fucci, L. Fulton, S. Gallorini, L. Gatignon, A. Gianoli, S. Giudici, L. Glonti, A. Goncalves Martins, F. Gonnella, E. Goudzovski, R. Guida, E. Gushchin, F. Hahn, B. Hallgren, H. Heath, F. Herman, D. Hutchcroft, E. Iacopini, O. Jamet, P. Jarron, K. Kampf, J. Kaplon, V. Karjavin, V. Kekelidze, S. Kholodenko, G. Khoriauli, A. Khudyakov, Yu. Kiryushin, K. Kleinknecht, A. Kluge, M. Koval, V. Kozhuharov, M. Krivda, Y. Kudenko, J. Kunze, G. Lamanna, C. Lazzeroni, R. Leitner, R. Lenci, M. Lenti, E. Leonardi, P. Lichard, R. Lietava, L. Litov, D. Lomidze, A. Lonardo, N. Lurkin, D. Madigozhin, G. Maire, A. Makarov, I. Mannelli, G. Mannocchi, A. Mapelli, F. Marchetto, P. Massarotti, K. Massri, P. Matak, G. Mazza, E. Menichetti, M. Mirra, M. Misheva, N. Molokanova, J. Morant, M. Morel, M. Moulson, S. Movchan, D. Munday, M. Napolitano, F. Newson, A. Norton, M. Noy, G. Nuessle, V. Obraztsov, S. Padolski, R. Page, V. Palladino, A. Pardons, E. Pedreschi, M. Pepe, F. Perez Gomez, M. Perrin-Terrin, P. Petrov, F. Petrucci, R. Piandani, M. Piccini, D. Pietreanu, J. Pinzino, M. Pivanti, I. Polenkevich, I. Popov, Yu. Potrebenikov, D. Protopopescu, F. Raffaelli, M. Raggi, P. Riedler, A. Romano, P. Rubin, G. Ruggiero, V. Russo, V. Ryjov, A. Salamon, G. Salina, V. Samsonov, E. Santovetti, G. Saracino, F. Sargeni, S. Schifano, V. Semenov, A. Sergi, M. Serra, S. Shkarovskiy, A. Sotnikov, V. Sougonyaev, M. Sozzi, T. Spadaro, F. Spinella, R. Staley, M. Statera, P. Sutcliffe, N. Szilasi, D. Tagnani, M. Valdata-Nappi, P. Valente, M. Vasile, V. Vassilieva, B. Velghe, M. Veltri, S. Venditti, M. Vormstein, H. Wahl, R. Wanke, P. Wertelaers, A. Winhart, R. Winston, B. Wrona, O. Yushchenko, M. Zamkovsky, A. Zinchenko.
} 


\section{Kaon physics at CERN}

The European Organization for Nuclear Research (CERN) has a long history of kaon decay in flight experiments. This proceedings will focus on the three most recent experiments, each of which was designed to perform detailed studies of charged kaon decays. The NA48/2 experiment recorded $\sim 10^{11}$ kaon decays during 2003 and 2004. After 2004, the experimental setup was modified and became the NA62-RK experiment. The NA62-RK experiment was specifically designed to measure the ratio of the kaon leptonic decay rates $\left(\mathrm{R}_{\mathrm{K}}\right)$. During 2007 more than $10^{10}$ events were recorded for this purpose.

The NA62 experiment begins taking data in October 2014. The primary physics goal of the NA62 experiment is to measure the $\mathrm{K}^{+} \rightarrow \pi^{+} v \bar{v}$ decay rate, however, a huge variety of rare and forbidden decays can be studied alongside.

In section 2 the NA48/2 and NA62-RK experimental setups are outlined. In section 2.1, a precise measurement of $R_{K}$ is described. In sections 2.2 and 2.3 searches for heavy neutrinos and a dark photon are discussed. In section 3 the NA62 experimental setup is outlined. The NA62 L0 trigger and expected physics performance are detailed in sections 3.1 and 3.2 respectively.

\section{The NA48/2 and NA62-RK experiments}

The NA48/2 and NA62-RK experiments both relied on beams of positive and negative secondary hadrons produced by impinging protons extracted from the CERN SPS onto a beryllium target. The beams were momentum selected, then propagated to a fiducial volume contained in a $114 \mathrm{~m}$ long cylindrical vacuum tank called the 'decay volume'. In the NA48/2 experiment, the secondary hadrons were selected to have a momentum of $(60 \pm 3) \mathrm{GeV} / c$, while for the NA62-RK experiment their momentum was $(74 \pm 1.4) \mathrm{GeV} / c$. The individual beam particles were not tagged, and their momenta were not measured. The hadron beams were accompanied by an intense flux of muons travelling outside the beam vacuum pipe.

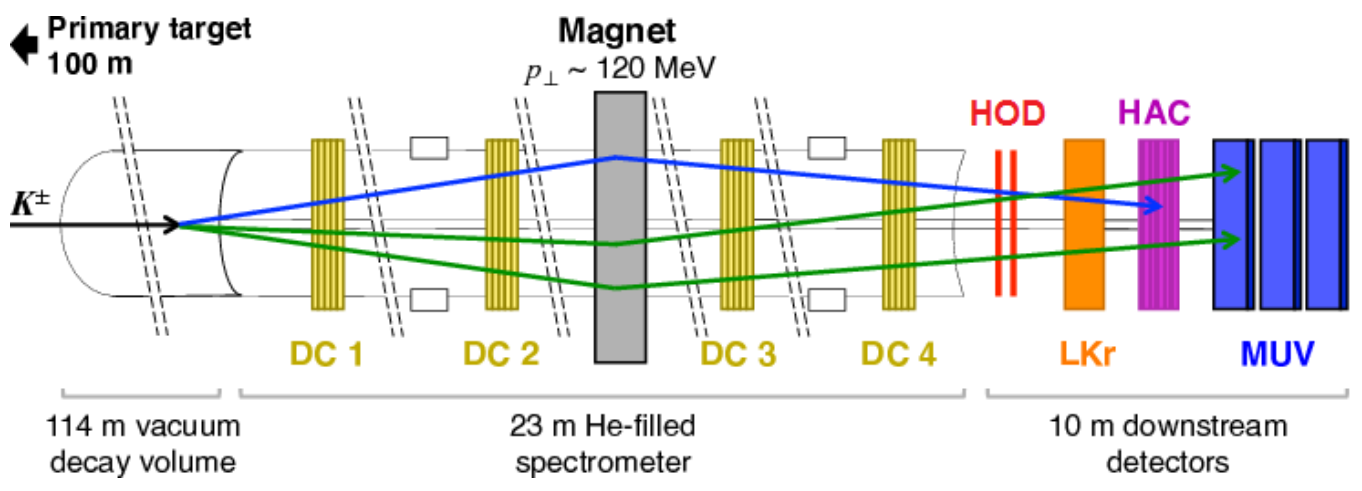

Figure 1: Sketch of the NA48/2 detector.

A sketch of the NA48/2 detector is shown in Fig. 1. The momenta of charged particles were measured by a magnetic spectrometer placed downstream of the decay volume. The spectrometer comprised four drift chambers (DCHs) and a dipole magnet located between the second and the third DCH which gave a horizontal transverse momentum kick to charged particles. In the NA48/2 
experiment the momentum kick of the magnet was $\sim 120 \mathrm{MeV} / c$, while for the NA62-RK experiment the momentum kick was $\sim 265 \mathrm{MeV} / c$. A counter hodoscope (HOD) consisting of two planes of orthogonal plastic scintillator strips producing fast charged particle trigger signals was placed after the spectrometer. A $127 \mathrm{~cm}\left(27 X_{0}\right)$ thick liquid krypton (LKr) electromagnetic calorimeter, used for lepton identification and as a photon veto detector, was located further downstream. A hadron calorimeter (HAC) and muon-veto system (MUV) provided further pion and muon identification. A more detailed description of the beam line and detector can be found in Refs. [1] and [2].

\subsection{Precision measurement of $R_{K}$ at NA62-RK}

The decays of pseudoscalar mesons to light leptons are helicity suppressed in the Standard Model (SM). The SM width of $K^{ \pm} \rightarrow \ell^{ \pm} v$ decays $\left(K_{\ell v}\right)$ is

$$
\Gamma^{\mathrm{SM}}\left(K^{ \pm} \rightarrow \ell^{ \pm} v\right)=\frac{G_{F}^{2} M_{K} M_{\ell}^{2}}{8 \pi}\left(1-\frac{M_{\ell}^{2}}{M_{K}^{2}}\right)^{2} f_{K}^{2}\left|V_{q q^{\prime}}\right|^{2},
$$

where $G_{F}^{2}$ is the Fermi constant, $M_{K}$ and $M_{\ell}$ are the kaon and lepton masses, $f_{K}$ is the kaon decay constant, and $V_{q q^{\prime}}$ is the corresponding CKM matrix element. Although the SM predictions for $K_{\ell v}$ decay rates are affected by hadronic uncertainties on the decay constant, ratios of decay rates of the kaon do not depend on $f_{K}$ and can be computed very precisely. In particular, the SM prediction for $\mathrm{R}_{\mathrm{K}}$ is $\mathrm{R}_{\mathrm{K}}^{\mathrm{SM}}=\Gamma^{\mathrm{SM}}\left(K_{e v}\right) / \Gamma^{\mathrm{SM}}\left(K_{\mu v}\right)=(2.477 \pm 0.001) \times 10^{-5}[3]$.

In extensions of the SM involving two Higgs doublets, $\mathrm{R}_{\mathrm{K}}$ is sensitive to lepton flavour violating effects induced by loop processes in which a charged Higgs boson $\left(H^{ \pm}\right)$is exchanged [4]. In such models $\mathrm{R}_{\mathrm{K}}$ can be enhanced by $\sim 1 \%$ [5], however, such enhancements are constrained by observables such as the $B_{S} \rightarrow \mu^{+} \mu^{-}$and $B^{+} \rightarrow \tau^{+} \nu$ decay rates [6].

The measurement of $\mathrm{R}_{\mathrm{K}}$ by the NA62-RK experiment utilised a relatively low beam intensity so that a highly efficient trigger could be used. The $K_{e v}$ trigger condition required the coincidence of signals in the two HOD planes, loose lower and upper limits on the DCH hit multiplicity and a $\mathrm{LKr}$ energy deposit of at least $10 \mathrm{GeV}$. The $K_{\mu \nu}$ trigger condition required a coincidence of the same HOD and DCH signals, and was downscaled by a factor of 150 .

Kinematic identification of the $K_{\mu \nu}$ decays was based on the reconstructed squared missing mass assuming the track to be an electron or a muon $\left(M_{\text {miss }}^{2}(\ell)\right)$. Lepton identification was based on the ratio of energy deposition in the $\mathrm{LKr}$ to momentum measured by the spectrometer $(E / p)$, as illustrated in Fig. 2a. The total $K_{e v}$ sample consisted of 145958 candidates with an estimated background of $(10.95 \pm 0.27) \%$, see Fig. $2 \mathrm{~b}$, while the $K_{\mu \nu}$ sample consisted of $4.282 \times 10^{7}$ candidates.

Using the total sample of kaon decays $R_{K}$ was measured to be $R_{K}=(2.488 \pm 0.010) \times 10^{-5}$, corresponding to a relative uncertainty of $\sim 0.4 \%$ [7]. The difference of the measured value from the SM prediction is $\Delta_{\mathrm{R}}=0.011 \pm 0.011$. The result is consistent with the SM expectation, and the earlier measurement by the KLOE experiment [8].

\subsection{Searches for heavy neutral leptons with kaon decays}

In the canonical SM the neutrinos are exactly massless and cannot oscillate, a property that is in direct contradiction to experimental evidence. One extension of the SM that is consistent 


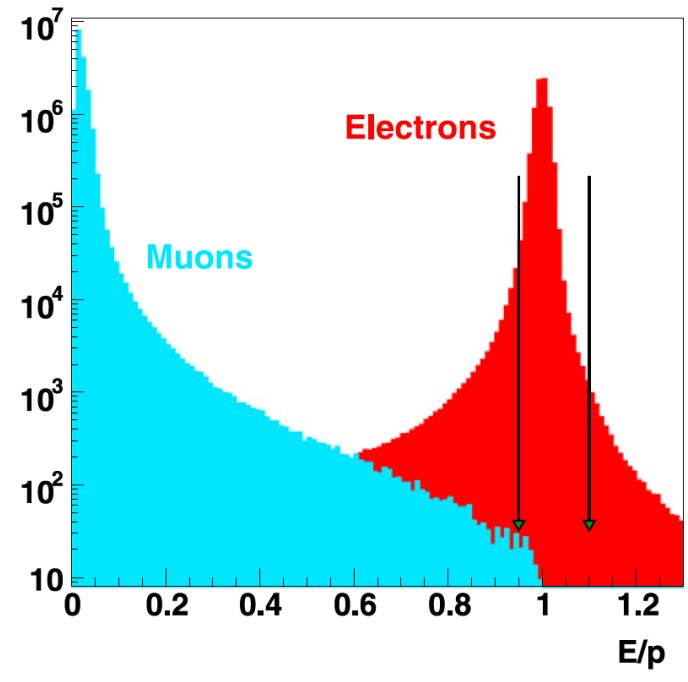

(a)

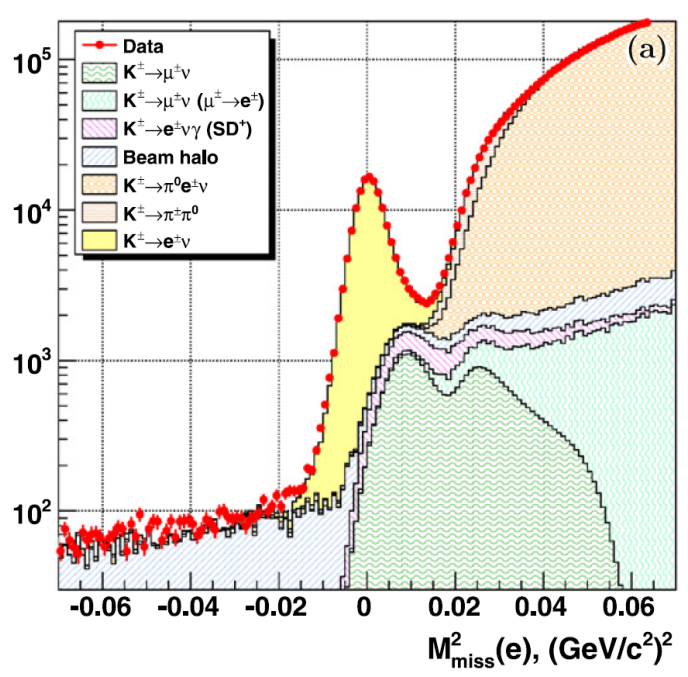

(b)

Figure 2: (a) Lepton identification criteria $E / p$ for simulated electrons (red filled area) and muons (blue filled area). (b) Missing mass spectrum, with data (red points), simulated $K_{e v}$ events (yellow filled area) and simulated background events (other filled areas).

with neutrino experiments, the so-called " $v$ MSM", introduces three right-handed singlet neutrinos $N_{I}(I=1-3)$ with three Majorana mass terms. The $v$ MSM can simultaneously explain neutrino oscillations, provides a cold dark matter candidate, and can reproduce the measured baryon asymmetry of the universe $[9,10]$.

The heavy neutrinos $N_{I}$ can be produced in charged kaon decays as long as the heavy neutrino mass $m_{N}$ is on the order of a few $\sim 100 \mathrm{MeV}$. Searches for heavy neutrinos are performed at CERN, however, two distinct analyses must be performed. The two analyses correspond to the situations where: the neutrino lifetime is long, and the neutrino decay vertex is distinct from that of the kaon; or the neutrino lifetime is short, and the neutrino decay vertex is indistinguishable from that of the kaon. An NA62-RK search for heavy neutrinos with long lifetimes is currently under way, performed by seeking the $K^{+} \rightarrow \mu^{+} N_{I}$ decay. Although topologically similar to $K_{\mu \nu}$, kaon decays to the heavy neutrino will peak at large values of $M_{\text {miss }}^{2}(\mu)$, see Fig. 3 .

The NA48/2 experiment performed a search for a short-lived heavy neutrino in the decay $K^{+} \rightarrow \mu^{+} \mu^{+} \pi^{-}\left(K_{\mu \mu \pi}\right)$, which is forbidden in the SM but can be mediated by heavy neutrinos decaying to $N_{I} \rightarrow \mu^{+} \pi^{-}$. In NA48/2 the kaon and heavy neutrino decay vertices are indistinguishable if the heavy neutrino lifetime is less than $\sim 10 \mathrm{ps}$. A large background from $K^{+} \rightarrow \pi^{+} \pi^{-} \pi^{+}$ decays is expected due to pion decays in flight. The analysis utilised the $K^{+} \rightarrow \pi^{+} \mu^{+} \mu^{-}$decay as a control channel, see Fig. 4a.

The heavy neutrino mediated decay $K_{\mu \mu \pi}$ would appear as an peak of events in the $\mu^{+} \mu^{+} \pi^{-}$ invariant mass $\left(M_{\mu \mu \pi}\right)$. Upon inspection of the $M_{\mu \mu \pi}$ spectrum, the number of events in a region around the nominal kaon mass was consistent with the number expected from background, see Fig. 4b. Since no excess was observed, a limit was set on the $K_{\mu \mu \pi}$ branching fraction at less than $1.1 \times 10^{-9}$ [11], which is the most precise limit on the $K_{\mu \mu \pi}$ branching fraction to date. 


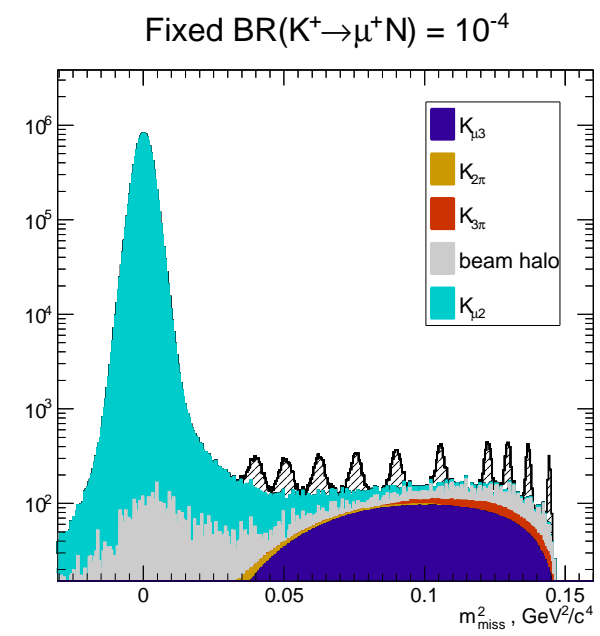

Figure 3: Squared missing mass spectrum for the NA62-RK heavy neutrino search, with the various background sources (filled areas) and various excesses due to a heavy neutrino assuming a $K^{+} \rightarrow \mu^{+} N_{I}$ branching fraction of $10^{-4}$ (black striped histogram). The position of the excess is determined by the mass of the heavy neutrino.

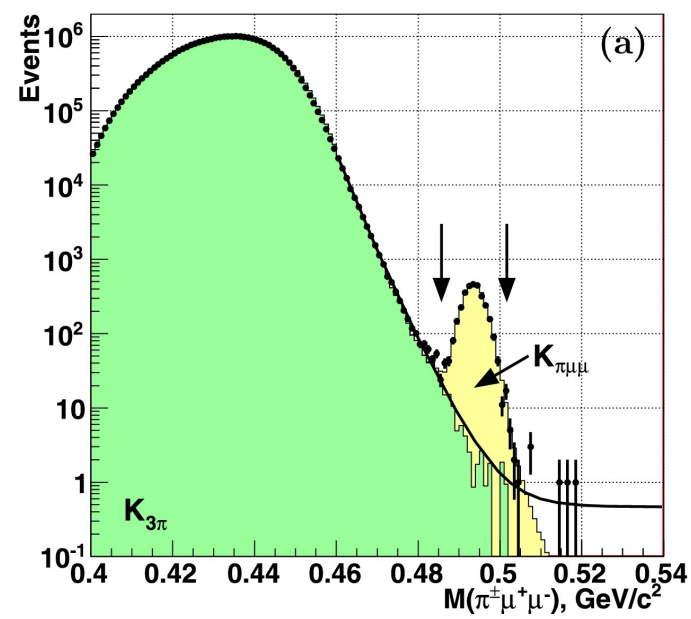

(a)

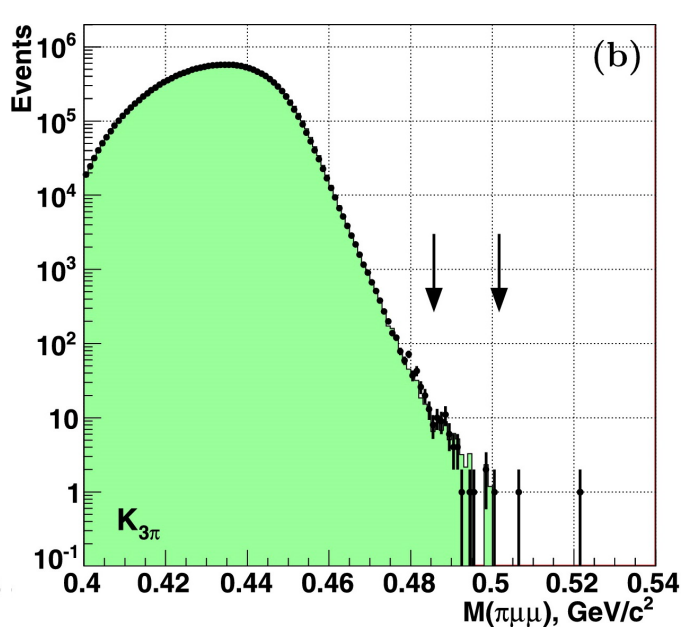

(b)

Figure 4: Reconstructed mass spectra of (a) $K^{+} \rightarrow \pi^{+} \mu^{+} \mu^{-}$and (b) $K^{+} \rightarrow \mu^{+} \mu^{+} \pi^{-}$candidates. The data (dots) are shown with simulated $K^{+} \rightarrow \pi^{+} \pi^{-} \pi^{+}$(green filled area) and $K^{+} \rightarrow \pi^{+} \mu^{+} \mu^{-}$(yellow filled area) events. In (a), an empirical fit to the $K^{+} \rightarrow \pi^{+} \pi^{-} \pi^{+}$background is overlaid. The standard signal region is indicated with arrows.

\subsection{Searches for dark photons with kaon decays}

Powerful evidence for new physics comes from the existence of dark matter. Dark matter dominates the matter density in our Universe, but very little is known about it. Its existence may hint toward the existence of a dark sector, which could include a rich structure of particles that do not interact with the known strong, weak, or electromagnetic forces. There are only a few interactions allowed by SM symmetries that can communicate between the SM sector and the dark sector. One example is the dark photon, which arises by introducing an extra U(1) gauge symmetry 
to the SM [12].

The dark photon can have a substantial contribution to the $\pi^{0} \rightarrow \gamma e^{+} e^{-}$decay as it mixes with the SM photon. An NA48/2 analysis of the $\pi^{0} \rightarrow \gamma e^{+} e^{-}$decay is proceeding, utilising the $K^{+} \rightarrow \pi^{+} \pi^{0}$ decay to isolate a huge number of neutral pion decays. The $\pi^{0} \rightarrow \gamma e^{+} e^{-}$decay is not forbidden in the SM, but the dieletron pairs have broad spectrum of invariant masses $M_{e e}$. The NA48/2 analysis seeks a peak in the $M_{e e}$ spectrum consistent with the production of a dark photon, see Fig. 5.

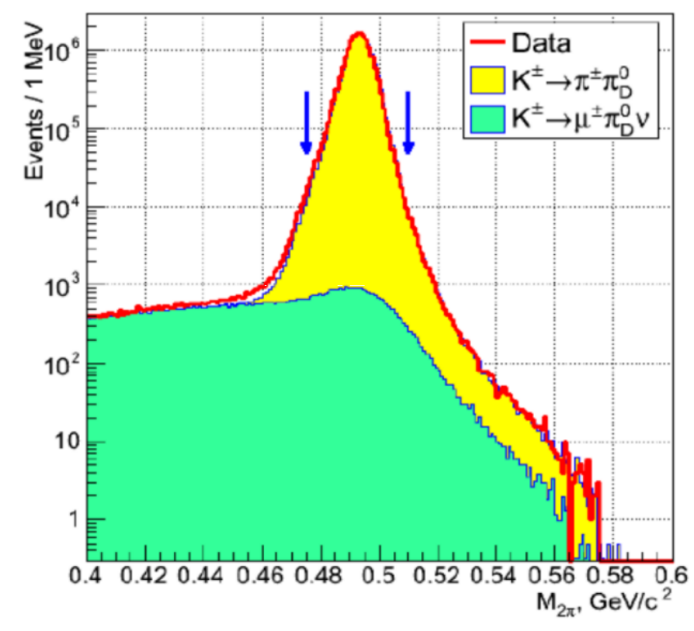

(a)

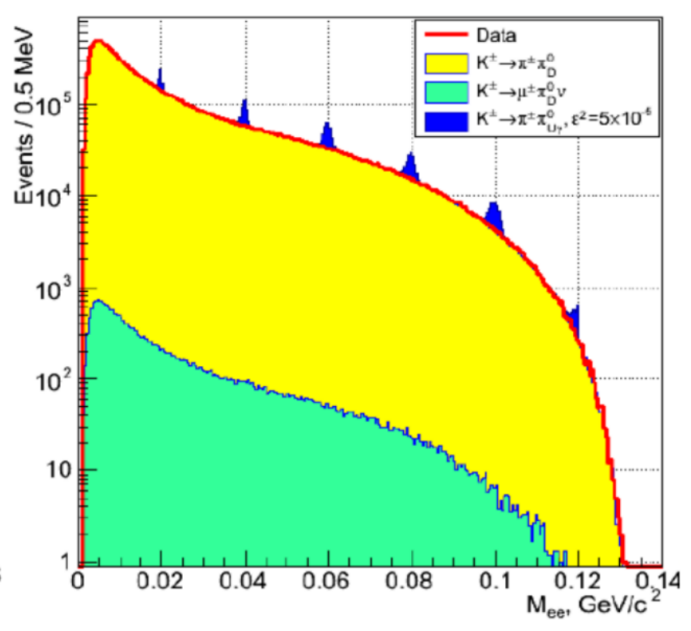

(b)

Figure 5: The reconstructed $\pi^{+} \pi^{0}$ (a) and $e^{+} e^{-}$(b) mass spectra of $\pi^{0} \rightarrow \gamma e^{+} e^{-}$candidates in NA48/2 data (dots), overlaid with simulated: $K^{+} \rightarrow \pi^{+} \pi^{0}$ events (yellow filled area); $K^{+} \rightarrow \mu^{+} \pi^{0} v$ events (green filled area); and a number of expected signatures from decays mediated by a dark photon of various masses (blue filled area). In (a) the signal region is indicated with arrows.

\section{The NA62 experiment}

The primary aim of the NA62 experiment is to collect $\sim 100 \mathrm{~K}^{+} \rightarrow \pi^{+} v \bar{v}$ events, over two years of data taking, with a signal-to-background ratio of 10:1. A sketch of the detector system is shown in Fig. 6. The NA62 experiment uses the same positive hadron beam as the NA62RK experiment. At NA62 however the beam particles, of which about $6 \%$ are kaons, will pass through a CEDAR volume and kaons will be tagged by the KTAG. The beam then passes through a silicon pixel detector called the GigaTracker, which measures the momentum of each particle in the beam, and then into an $80 \mathrm{~m}$ long fiducial volume inside a vacuum vessel. Charged particles outside of the beam are measured in a spectrometer comprised of four straw chambers, two before and two after a dipole magnet (the same as used in the NA48/2 experiment) that gives charged particles a transverse momentum kick of $\sim 270 \mathrm{MeV}$. A hermetic photon veto system is used to reject events with unwanted photons. The photon vetoes include the large angle veto (LAV), the NA48/2 LKr, and calorimeters at the end of the beam pipe. The separation of charged pions and muons is performed by the RICH detector, the $\mathrm{LKr}$ calorimeter and a new three-layer muon veto detector (MUV1-3). The MUV3 part is a segmented layer of plastic scintillator situated behind an 


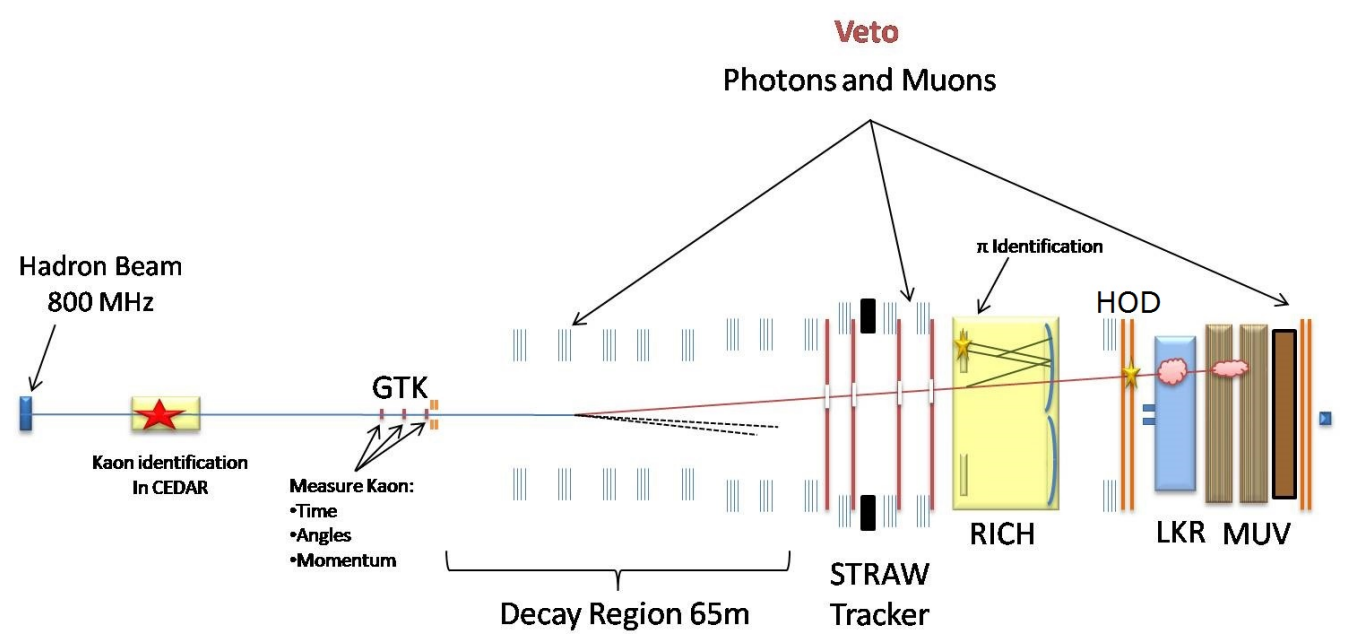

Figure 6: Sketch of the NA62 detector.

iron wall, and has a response time fast enough for it to be used for triggering. The NA48/2 HOD is situated after the RICH detector and is used for triggering on charged particles. A more detailed description of the NA62 experiment can be found in Ref. [13].

\subsection{The NA62 L0 trigger}

The unprecedented number of charged kaon decays expected to occur in the NA62 fiducial volume will potentially lead to record sensitivities for a wide range of rare and forbidden kaon decays. Moreover, the $K^{+} \rightarrow \pi^{+} \pi^{0}$ decay will provide a huge sample of tagged $\pi^{0}$ decays in vacuum, making NA62 a unique " $\pi^{0}$ factory". The exploitation of rare and forbidden decays alongside $K^{+} \rightarrow \pi^{+} v \bar{v}$ will rely on dedicated trigger chains.

The NA62 trigger is divided into three logical levels. The first is a hardware L0 trigger that reduces the $\sim 10 \mathrm{MHz}$ input rate to $\sim 1 \mathrm{MHz}$ using input from a few subdetectors. The remaining stages, L1 and L2, are implemented as a $\mathrm{C}++$ program running on a PC farm. The L1 stage reduces the $\sim 1 \mathrm{MHz}$ input rate to $\sim 100 \mathrm{kHz}$ using information computed independently by each sub-detector. The L2 stage reduces the $\sim 100 \mathrm{kHz}$ input rate to $\sim 4 \mathrm{kHz}$, which can be written to permanent storage, using reconstructed events.

A bandwidth of $\sim 400 \mathrm{kHz}$ will be available to collect rare and forbidden events. The L0 trigger relies on information from the RICH, CHOD, MUV3 and LKr subdetectors. The quantities used in the L0 trigger, known as primitives, are summarised as follows: a coincidence of signals in at least $N$ RICH super-cells $\left(R_{N}\right)$; a coincidence in the two CHOD planes in at least $N$ quadrants $\left(Q_{N}\right)$; a coincidence in the two CHOD planes in one of the two pairs of opposite quadrants $\left(Q_{X}\right)$; a coincidence in at least $N$ MUV3 pads that are not adjacent to the beam pipe hole $\left(M O_{N}\right)$; a total LKr energy deposit of at least $x \mathrm{GeV}\left(E_{x}\right)$.

The primitives are combined by the $\mathrm{L} 0$ trigger processor to form six classes of $\mathrm{L} 0$ trigger. The single-track trigger requires $R_{4} * Q_{1}$, where the asterisk indicates a logical AND, while the multitrack trigger requires $R_{10} * Q_{X}$. The 'single track with electromagnetic energy' trigger is defined using the single-track trigger with the addition of the $E_{10}$ primitive. Dilepton triggers are defined 
using the multi-track trigger with the addition of either: the $E_{10}$ primitive for the 'dielectron' trigger; or the $\mathrm{MO}_{2}$ primitive for the 'dimuon' trigger. The 'multi-track with muon and electromagnetic energy' trigger is defined using the multi-track trigger with the addition of the $M_{1}$ and $E_{10}$ primitives. The total event rate of the trigger scheme outlined above is expected to be $\sim 400 \mathrm{kHz}$, consistent with the constraint imposed by the maximum L0 trigger output rate.

\subsection{Physics prospects of the NA62 experiment}

The NA62 experiment will collect around $\sim 10^{13}$ charged kaon decays in two years of nominal data taking. As $\sim 10 \%$ of the decays will fall within the NA62 acceptance, limits on rare and forbidden decays can be set at the $\sim 10^{-12}$ level. Limits at this level are generally orders of magnitude more stringent than current limits. For decays of neutral pions limits at $\sim 10^{-10}$ can be set. The neutral pion limits are less stringent since only $\sim 10^{12}$ neutral pion decays are expected from $K^{+} \rightarrow \pi^{+} \pi^{0}$ decays, and the acceptance for these decays is typically $\sim 1 \%$.

An $R_{K}$ measurement at NA62 will benefit considerably from the enhanced separation of charged pions and muons provided by the RICH detector. Furthermore, the hermetic photon veto and precise timing of NA62, which enables the matching of particles in the detector to the decay of a specific kaon in the beam, will considerably reduce the number of background events. In total, the collected $K_{e v}$ sample expected at NA62 will be about ten times more pure than that collected by NA62-RK. This will lead to an improvement in the measurement by more than a factor of two.

A search for short-lived heavy neutrinos in the $K^{+} \rightarrow \mu^{+} \mu^{+} \pi^{-}$decay will benefit from the larger transverse momentum kick from the NA62 dipole magnet compared to the NA48/2 analysis. At NA62 the $K^{+} \rightarrow \pi^{+} \pi^{-} \pi^{+}$background should be reduced to a negligible level. This reduction is complimented by an expected improvement, of more than a factor two, in the $\mu^{+} \mu^{+} \pi^{-}$mass resolution.

\section{References}

[1] J. R. Batley, et al., Eur. Phys. J. C 52 (2007) 875.

[2] V. Fanti, et al., Nucl. Inst. Methods A 574 (2007) 433.

[3] V. Cirigliano, I. Rosell, Phys. Rev. Lett. 99 (2007) 231801.

[4] A. Masiero, P. Paradisi, R. Petronzio, Phys. Rev. D 74 (2006) 011701;

A. Masiero, P. Paradisi, R. Petronzio, JHEP 0811 (2008) 042.

[5] J. Girrbach, U. Nierste, arXiv:1202.4906.

[6] R. M. Fonseca, J. C. Romão, A. M. Teixeira, Eur. Phys. J. C 72 (2012) 2228.

[7] C. Lazzeroni, et al., Phys. Lett. B 719 (2013) 326-336.

[8] F. Ambrosino, et al., Eur. Phys. J. C 64 (2009) 627.

[9] T. Asaka and M. Shaposhnikov, Phys. Lett. B 620 (2005) 17-26.

[10] T. Asaka, S. Blanchet and M. Shaposhnikov, Phys. Lett. B 631 (2005) 151-156.

[11] J. R. Batley, et al., Phys. Lett. B 697 (2011) 107.

[12] R. Essig, et al., arXiv:1311.0029.

[13] The NA62 collaboration, NA62-10-07, https://cds.cern.ch/record/1404985. 